\title{
The CANHEART health index: a tool for monitoring the cardiovascular health of the Canadian population
}

\author{
Laura C. Maclagan MSc, Jungwee Park PhD, Claudia Sanmartin PhD, Karan R. Mathur HBSc, Doug Roth MBA, \\ Douglas G. Manuel MD MSc, Andrea Gershon MD MSc, Gillian L. Booth MD MSc, Sacha Bhatia MD MBA, \\ Clare L. Atzema MD MSc, Jack V. Tu MD PhD
}

Competing interests: None declared.

This article has been peer reviewed.

Correspondence to: JackTu, tu@ices.on.ca

CMAJ 2014. DOI:10.1503 /cmaj.131358

\section{Abstract}

Background: To comprehensively examine the cardiovascular health of Canadians, we developed the Cardiovascular Health in Ambulatory Care Research Team (CANHEART) health index. We analyzed trends in health behaviours and factors to monitor the cardiovascular health of the Canadian population.

Methods: We used data from the Canadian Community Health Survey (2003-2011 [excluding 2005]; response rates $70 \%-81 \%$ ) to examine trends in the prevalence of 6 cardiovascular health factors and behaviours (smoking, physical activity, fruit and vegetable consumption, overweight/obesity, diabetes and hypertension) among Canadian adults aged 20 or older. We defined ideal criteria for each of the 6 health metrics. The number of ideal metrics was summed to create the CANHEART health index; values range from 0 (worst) to 6 (best or ideal). A separate CANHEART index was developed for youth age 12-19 years; this index included 4 health factors and behaviours (smoking, physical activity, fruit and vegetable consumption and overweight/obesity). We determined the prevalence of ideal cardiovascular health and the mean CANHEART health index score, stratified by age, sex and province.
Results: During the study period, physical activity and fruit and vegetable consumption increased and smoking decreased among Canadian adults. The prevalence of overweight/obesity, hypertension and diabetes increased. In 2009-2010, 9.4\% of Canadian adults were in ideal cardiovascular health, $53.3 \%$ were in intermediate health (4-5 healthy factors or behaviours), and $37.3 \%$ were in poor cardiovascular health (0-3 healthy factors or behaviours). Twice as many women as men were in ideal cardiovascular health $(12.8 \%$ vs. $6.1 \%$ ). Among youth, the prevalence of smoking decreased and the prevalence of overweight/obesity increased. In 2009-2010, 16.6\% of Canadian youth were in ideal cardiovascular health, $33.7 \%$ were in intermediate health (3 healthy factors or behaviours), and $49.7 \%$ were in poor cardiovascular health (0-2 healthy factors or behaviours).

Interpretation: Fewer than 1 in 10 Canadian adults and 1 in 5 Canadian youth were in ideal cardiovascular health from 2003 to 2011. Intensive health promotion activities are needed to meet the Heart and Stroke Foundation of Canada's goal of improving the cardiovascular health of Canadians by $10 \%$ by 2020 as measured by the CANHEART health index.
$\mathrm{C}$ ardiovascular disease, which includes heart disease and stroke, is the second leading cause of death in Canada and is associated with disability, low quality of life and high economic costs. ${ }^{1}$ Although much previous research has focused on the contribution of individual health behaviours and factors to the risk of cardiovascular disease, there has been little focus on comprehensively examining the cardiovascular health of the population.

In 2010, the American Heart Association introduced a definition of ideal cardiovascular health based on 7 health factors and behaviours (smok- ing, body mass index [BMI], diet, physical activity, and blood pressure, glucose and cholesterol levels). ${ }^{2}$ Criteria were established to define ideal levels of each health factor or behaviour. The American Heart Association set a goal of improving the cardiovascular health of Americans by $20 \%$ by 2020 . Studies have shown that meeting 6-7 metrics of ideal cardiovascular health is associated with an $89 \%$ lower incidence of cardiovascular disease, ${ }^{3} 76 \%$ reduction in stroke incidence, ${ }^{4}$ $70 \%$ reduction in ischemic heart disease mortality $^{5}$ and $51 \%$ lower incidence of cancer ${ }^{6}$ compared with meeting 0 or 1 of the ideal metrics. 
We worked with the Heart and Stroke Foundation of Canada to measure trends in the prevalence of health behaviours and factors in the Canadian population and to develop a definition of ideal cardiovascular health. We developed the Cardiovascular Health in Ambulatory Care Research Team (CANHEART) health index as an exploratory approach to measure cardiovascu- lar health. We examined the temporal and spatial trends in cardiovascular health factors and behaviours by age, sex and province.

\section{Methods}

This study was part of the CANHEART research initiative (www.canheart.ca), which is aimed at

Table 1: Definition of ideal cardiovascular health among Canadian adults* and youth $\dagger$

\begin{tabular}{|c|c|}
\hline Metric & Ideal cardiovascular health \\
\hline \multicolumn{2}{|c|}{ Smoking } \\
\hline Adults & $\begin{array}{l}\text { Nonsmoker or former daily or occasional smoker who quit more than } \\
12 \text { months ago }\end{array}$ \\
\hline Youth & Never tried smoking or never smoked a whole cigarette \\
\hline \multicolumn{2}{|c|}{ Overweight/obesity } \\
\hline Adults & $\mathrm{BMI}<25 \ddagger$ \\
\hline Youth & Age- and sex-specific BMI cut-points ${ }^{8} ;<25$ for youth aged $18-19 \mathrm{yr}$ \\
\hline \multicolumn{2}{|c|}{ Leisure physical activity } \\
\hline Adults & $\geq 1.5 \mathrm{kcal} / \mathrm{kg} / \mathrm{d}$ (equivalent to $\geq 30 \mathrm{~min}$ of walking per $\mathrm{d}$ ) \\
\hline Youth & $\begin{array}{l}\geq 3.0 \mathrm{kcal} / \mathrm{kg} / \mathrm{d} \text { (equivalent to } \geq 60 \mathrm{~min} \text { of walking or } 20 \mathrm{~min} \text { of running or } \\
\text { jogging per d) }\end{array}$ \\
\hline \multicolumn{2}{|c|}{ Fruit and vegetable consumption } \\
\hline Adults & Fruit and vegetables consumed $\geq 5$ times per $d$ \\
\hline Youth & Fruit and vegetables consumed $\geq 5$ times per $d$ \\
\hline \multicolumn{2}{|c|}{ Hypertension } \\
\hline Adults & Self-reported hypertension diagnosed by a health care professional \\
\hline \multicolumn{2}{|c|}{ Diabetes } \\
\hline Adults & Self-reported diabetes diagnosed by a health care professional \\
\hline $\begin{array}{l}\text { Note: BM } \\
\text { *Aged } 20 \\
\text { †Aged } 12 \\
\text { †People } \\
\text { body wei }\end{array}$ & $\begin{array}{l}\text { ulations (e.g., Chinese, South Asian) should consider using a BMI cut-off of }<23 \text { for ideal } \\
\text { World Health Organization. }{ }^{9}\end{array}$ \\
\hline
\end{tabular}

Table 2: Prevalence estimates of ideal, intermediate and poor cardiovascular health based on data from the Canadian Community Health Survey, 2009-2010

\begin{tabular}{|c|c|c|c|c|}
\hline \multirow[b]{2}{*}{ Group } & \multicolumn{3}{|c|}{ Prevalence, \% $(95 \% \mathrm{Cl})$} & \multirow{2}{*}{$\begin{array}{l}\text { CANHEART health } \\
\text { index score, mean } \\
(95 \% \mathrm{Cl})\end{array}$} \\
\hline & Poor health* & $\begin{array}{l}\text { Intermediate } \\
\text { healtht }\end{array}$ & Ideal health $\ddagger$ & \\
\hline \multicolumn{5}{|c|}{ Adults (> 20 yr) } \\
\hline Overall & $37.3(36.8-37.8)$ & $53.3(52.7-53.8)$ & $9.4 \quad(9.1-9.8)$ & 3.87 (3.86-3.89) \\
\hline Men & $42.0(41.2-42.8)$ & $51.9(51.1-52.7)$ & $6.1 \quad(5.7-6.5)$ & $3.71(3.69-3.73)$ \\
\hline Women & 32.6 (31.9-33.3) & $54.6(53.9-55.3)$ & $12.8(12.3-13.3)$ & $4.04(4.02-4.06)$ \\
\hline \multicolumn{5}{|c|}{ Youth (12-19 yr) } \\
\hline Overall & $49.7(48.4-51.0)$ & 33.7 (32.4-34.9) & $16.6(15.6-17.6)$ & $2.49(2.47-2.52)$ \\
\hline Boys & $49.9(48.1-51.6)$ & $34.0(32.3-35.8)$ & $16.1(14.7-17.5)$ & $2.48(2.45-2.52)$ \\
\hline Girls & $49.6(47.7-51.5)$ & $33.3(31.6-35.1)$ & $17.1(15.7-18.4)$ & $2.51(2.47-2.54)$ \\
\hline
\end{tabular}


measuring and improving the cardiovascular health and quality of ambulatory cardiovascular care in Canada. Ethics approval for this study was obtained from Sunnybrook Health Sciences Centre.

\section{Data sources and study population}

We used data from the Canadian Community Health Survey (CCHS), a cross-sectional telephone survey of self-reported health status, determinants and health service use of Canadians aged 12 and older. We included CCHS cycles 1.1 (2001), 2.1 (2003), 3.1 (2005), 4.1 (2007-2008), 2009-2010 and 2011. The CCHS uses a complex multistage cluster-sampling design to provide reliable estimates at the health region and provincial levels and covers $98 \%$ of the Canadian population.?
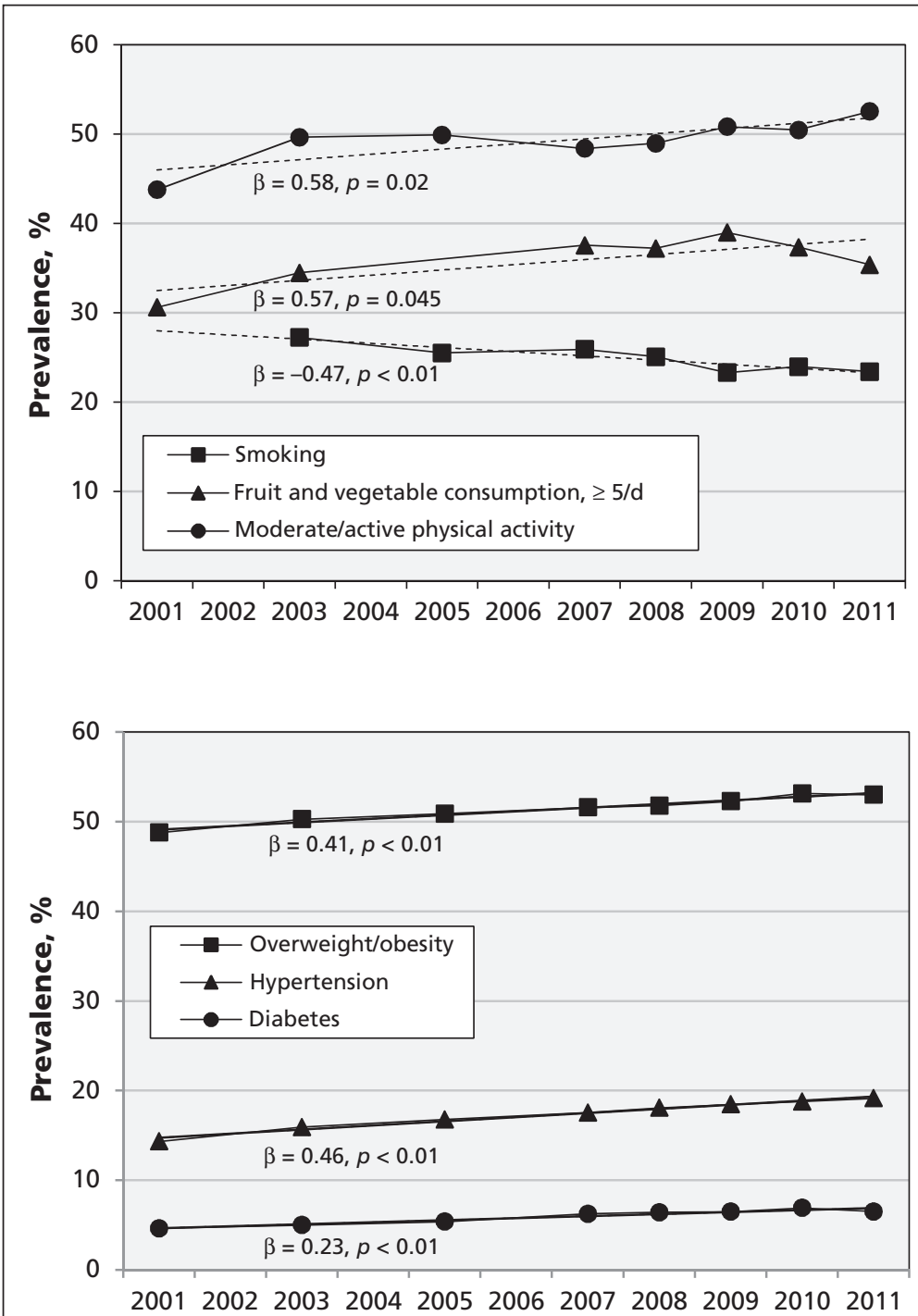

Figure 1: Trends in health factors and behaviours among Canadian adults aged 20 years and older. The beta $(\beta)$ coefficients can be interpreted as the annual percentage change in each factor. Dashed lines indicate the regression line. Source: Canadian Community Health Survey 2001-2011.
In this study, we calculated the CANHEART adult health index score for adults aged 20 and older. Youth aged 12-19 years were included in a separate CANHEART youth health index. We excluded pregnant women and children aged less than 12 years. We excluded 2 cycles of CCHS from the calculation of the CANHEART health index because the index requires the availability of all relevant health behaviour and factors (these cycles were retained for other analyses): the 2001 cycle lacked specific information about smoking cessation, and the 2005 cycle lacked information about fruit and vegetable consumption for all provinces. We chose the CCHS as the primary data source for the analysis of health behaviours and factors because of continuous data collection (allows analysis of historical and future trends) and the large sample size (allows calculation of the index at the health region and provincial levels).

\section{Study variables}

The criteria used to define ideal cardiovascular health in adults and youth are shown in Table 1. The definition of ideal cardiovascular health that we developed in this study was a modified version of the American Heart Association's definition of ideal cardiovascular health. ${ }^{2}$ Our definition takes the type of data available in Canada from the CCHS into consideration. Fruit and vegetable intake was based on the average number of times fruits and vegetables were consumed per day (including fruit juice, fruits, green salad, carrots, other vegetables; we excluded potatoes because of the fried and high-fat nature of some potato-based foods). We chose physical activity thresholds that aligned as closely as possible with the Canadian Physical Activity Guidelines. ${ }^{10}$ Body mass index was calculated using self-reported height and weight. Respondents self-reported hypertension and diabetes diagnosed by a health care professional. Information about cholesterol level was not available in the CCHS. We used age- and sex-specific BMI cutoffs from the International Obesity Task Force to define overweight/obesity among youth aged 12-17 years. ${ }^{8}$ We did not include hypertension and diabetes in the youth CANHEART health index because of the low prevalence of these conditions in this group.

\section{CANHEART health index}

We assigned the presence of each ideal health behaviour or factor a value of 1 for an ideal state and 0 for a nonideal state. These values were summed to create the CANHEART health index score; scores ranged from 0 (worst) to 6 (best) among adults and 0 (worst) to 4 (best) among 
youth. The cardiovascular health categories (Table 2) were chosen based on the prevalence of heart disease among individuals with each CANHEART score relative to the population average; scores with an above average prevalence of heart disease were categorized as poor cardiovascular health, and scores with a below average prevalence were categorized as intermediate and ideal cardiovascular health.

\section{Statistical analysis}

The prevalence of each health behaviour or factor in adults and youth was calculated by sex and year to examine temporal trends. We used simple linear regression models to assess trends in health behaviours and factors. We calculated beta coefficients and $p$ values for trends in each health behaviour or factor. We calculated the prevalence of CANHEART health index scores and categories (ideal, intermediate or poor) and the mean CANHEART health index score among adults and youth by sex, year and province. The prevalence of heart disease was calculated for individuals with each CANHEART health index score. We weighted the data using Statistics Canada's survey weights to generate estimates that were representative of the Canadian population. We calculated $95 \%$ confidence intervals (CIs) using bootstrapping methods.

\section{Results}

\section{Study population}

In the calculation of the CANHEART health index, we analyzed data for 464883 CCHS respondents (cycles for 2003-2011, excluding 2005). The survey response rates (\% of households selected for the survey for which an individual responded) ranged from $70 \%$ to $81 \%$. Data for 728657 respondents were included in the calculation of trends (fewer were included for the calculation of smoking and fruit and vegetable consumption trends because of data availability).

\section{Trends in health factors and behaviours}

Among Canadian adults, we found significant increasing trends for the prevalence of overweight/obesity, hypertension and diabetes, which increased at rates of $0.41 \%, 0.46 \%$ and $0.23 \%$ per year, respectively (Figure 1 ). There were significantly increasing trends for the prevalence of fruit and vegetable consumption ( $\geq 5$ times per day) and physical activity (about $0.5 \%$ per $y r)$; the prevalence of smoking decreased at about the same rate. Among youth, the prevalence of smoking decreased significantly at more than $1 \%$ per year (Figure 2 ). The prevalence of obesity/overweight increased at a rate of $0.23 \%$ per year; there were also increasing trends for physical activity and fruit and vegetable consumption; however, these results were not significant.

\section{Trends in cardiovascular health}

The prevalence of ideal cardiovascular health among Canadian adults remained stable during the study period (2003: $8.3 \%$ [95\% CI 8.0\%8.6\%]; 2011: $8.8 \%$ [95\% CI 8.3\%-9.2\%]). The mean adult CANHEART score was stable over this period (2003: 3.86 [95\% CI 3.85-3.87]; 2011: 3.86 [95\% CI 3.84-3.88]) (Appendix 1, available at www.cmaj.ca/lookup/suppl/doi:10 .1503/cmaj.131358/-/DC1).

Among youth, the prevalence of ideal cardiovascular health increased significantly from $13.1 \%$ in 2003 (95\% CI $12.4 \%-13.9 \%$ ) to $15.6 \%$

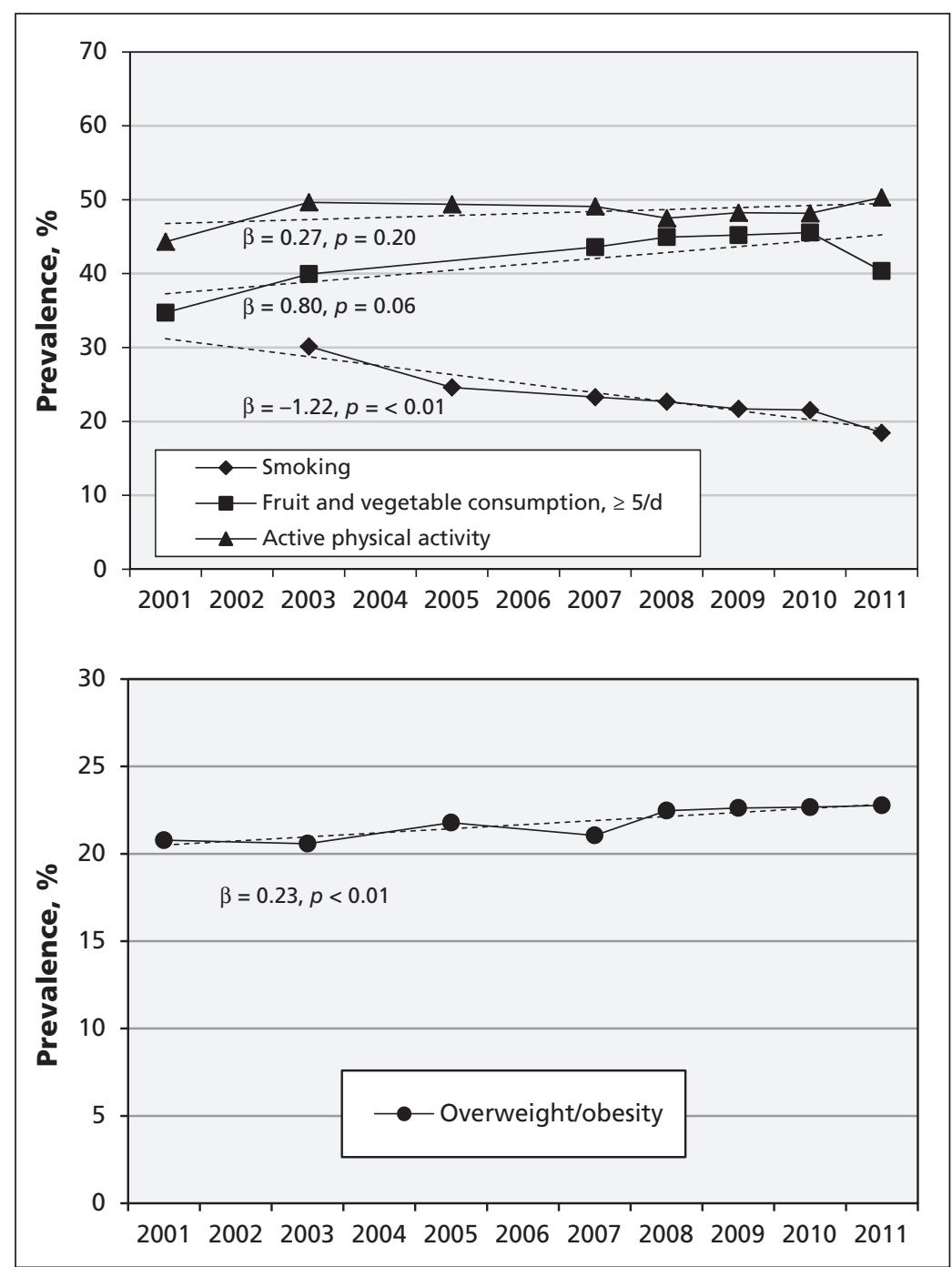

Figure 2: Trends in health behaviours and factors among Canadian youth aged $12-19$ years. The beta $(\beta)$ coefficients can be interpreted as the annual percentage change in each factor. Dashed lines indicate the regression line. Source: Canadian Community Health Survey 2001-2011. 
in 2011 (95\% CI 14.2\%-17.0\%). A significant increase was also observed for the mean CANHEART score in this age group (2003: 2.39 [95\% CI 2.37-2.41]; 2011: 2.49 [95\% CI 2.46-2.53]) (Appendix 1).

\section{Prevalence of ideal cardiovascular health} In 2009-2010, 9.4\% (95\% CI 9.1\%-9.8\%) of Canadian adults age 20 and older were in ideal cardiovascular health (Table 2). More than onethird of Canadian adults $(37.3 \%$ [95\% CI $36.8 \%-37.8 \%$ ]) were in poor cardiovascular health (0-3 ideal health factors or behaviours). Twice as many women $(12.8 \%$ [95\% CI $12.3 \%$ $13.3 \%])$ as men $(6.1 \%$ [95\% CI 5.7\%-6.5\%])

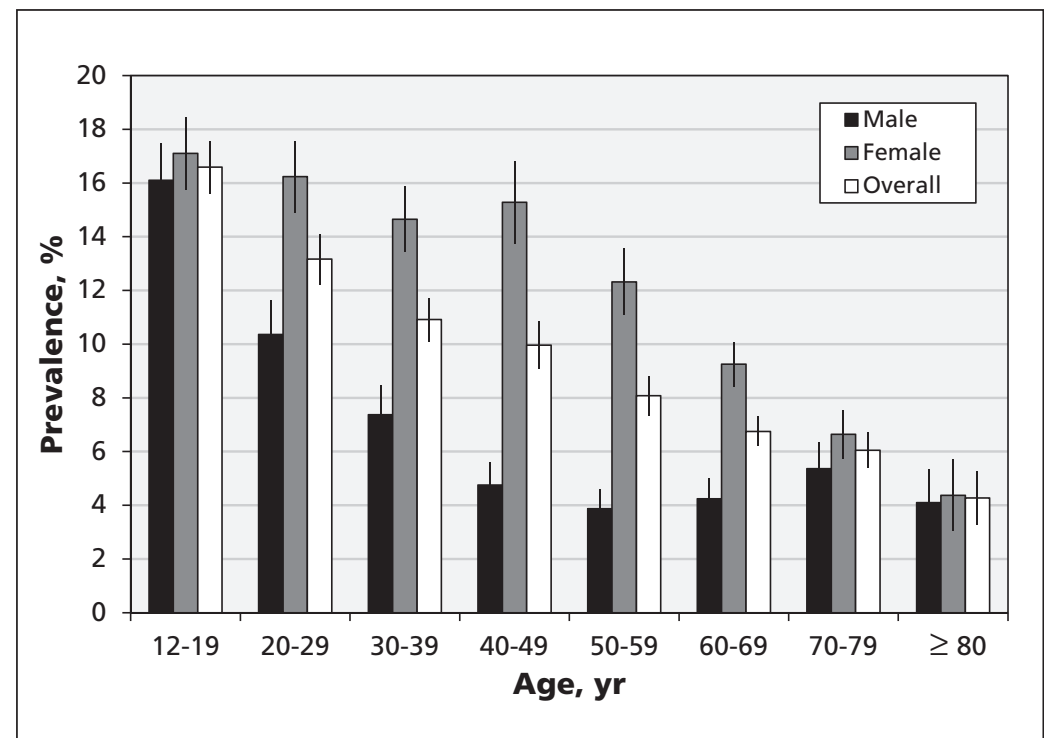

Figure 3: Prevalence of ideal cardiovascular health, by age group and sex. Error bars indicate $95 \%$ confidence intervals. Source: Canadian Community Health Survey 2009-2010.

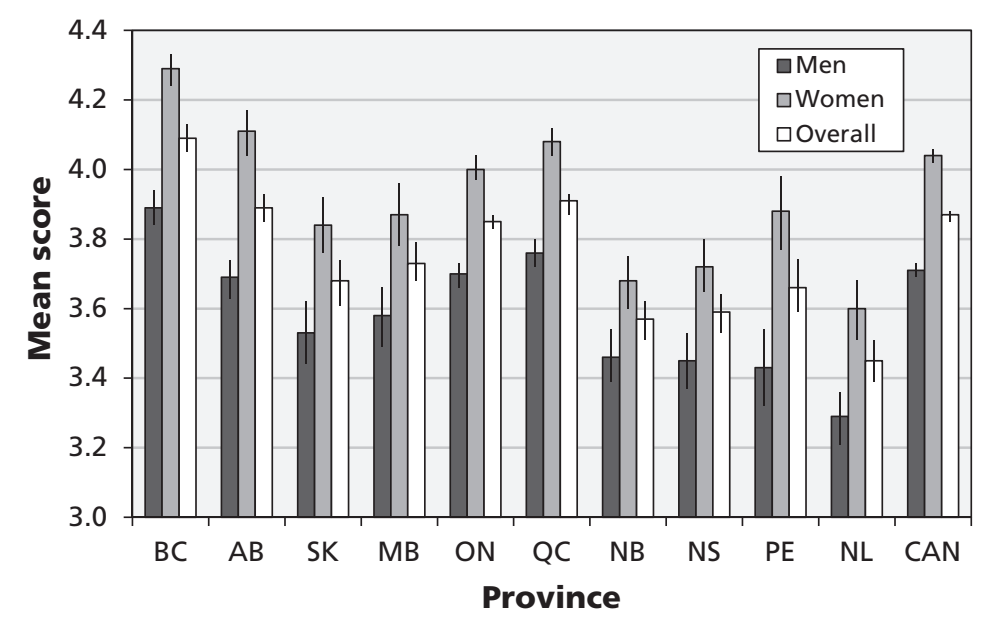

Figure 4: Mean CANHEART health index score among Canadian adults aged 20 years and older by province. Error bars indicate $95 \%$ confidence intervals. Source: Canadian Community Health Survey 2009-2010. were in ideal cardiovascular health. Women had a significantly higher mean CANHEART score and lower prevalence of poor cardiovascular health compared with men. In total, $16.6 \%$ of youth were in ideal cardiovascular health $(95 \%$ CI 15.6\%-17.6\%); however, almost half were in poor cardiovascular health $(49.7 \%$ [95\% CI $48.4 \%-51.0 \%]$ ). The proportion of youth in ideal, intermediate or poor cardiovascular health did not differ significantly by sex.

The prevalence of ideal cardiovascular health generally decreased with age among both men and women (Figure 3). The prevalence of ideal cardiovascular health was similar among men and women for the oldest and youngest age groups. The largest differences were observed in middle age: women aged 40-49 years had a 3 times greater prevalence of ideal cardiovascular health compared with men of the same age (15.3\% [95\% CI $13.8 \%-16.8 \%$ ] v. $4.8 \%$ [95\% CI $3.9 \%-5.6 \%$ ]).

The mean CANHEART score generally decreased from Western to Eastern provinces, from a high of 4.1 (95\% CI 4.1-4.1) in British Columbia to a low of 3.5 (95\% CI 3.4-3.5) in Newfoundland and Labrador (Figure 4). Ideal and intermediate cardiovascular health showed a similar pattern. The prevalence of poor cardiovascular health was lowest in British Columbia (30.1\% [95\% CI 28.8\%-31.5\%]) and highest in Newfoundland and Labrador $(51.7 \%$ [95\% CI $49.4 \%-54.0 \%]$ ).

\section{Prevalence of heart disease}

Adults in ideal cardiovascular health had a 16 times lower prevalence of heart disease compared with individuals with the worst cardiovascular health (score of 6: $1.6 \%$ [95\% CI 1.2\%-1.9\%]; score of 0: $25.2 \%$ [95\% CI 18.1\%-32.3\%]) (Figure 5). An increasing CANHEART score was correlated with a decreasing prevalence of heart disease $\left(R^{2}=0.89, p<0.001\right)$. Similar associations were found among men and women.

\section{Interpretation}

In this large Canadian population-based study, we found mixed trends in the prevalence of health factors and behaviours during the study period. Encouraging trends were found: among adults the prevalence of smoking decreased and the prevalence of fruit and vegetable consumption and physical activity increased, and the prevalence of smoking decreased among youth. However, the rising prevalence of overweight/ obesity among adults and youth and hypertension and diabetes among adults are cause for concern. Canadian population-based studies 
using other data sources have found similar increases in the prevalence of obesity, ${ }^{11}$ hypertension $^{12}$ and diabetes. ${ }^{13}$ The trends in risk factors and associated CANHEART health index scores should be closely monitored over time because these factors are associated with the development of many chronic diseases. ${ }^{14}$

From 2003 to 2011, the prevalence of ideal cardiovascular health and the mean CANHEART score were stable among adults and increased moderately among youth. However, decreasing trends in hospital admissions and death due to acute myocardial infarction, stroke and congestive heart failure during a similar period have previously been observed among Canadian adults..$^{15}$ These trends may be primarily because of better treatment of risk factors (e.g., cholesterol, diabetes, hypertension) and other advances in medical treatment as opposed to decreasing levels of risk factors. Also, the relative importance of each health behaviour and factor in the index on cardiovascular risk may not be equal (e.g., smoking v. fruit and vegetable intake). We elected to keep the index simple and easy to understand by weighting each factor equally, but additional studies should be conducted to determine whether variable weighting of each factor might improve the index; additional studies are also needed to validate the ability of the index to prospectively predict cardiovascular events.

In our study, Canadian women were in better cardiovascular health than men. Men had poorer health behaviours than women, except for physical activity, which was higher among men, and hypertension, which was similar between the sexes. There were fewer sex-related differences among youth. These findings corroborate evidence from other studies examining sex differences in cardiovascular risk factors, ${ }^{1,16}$ which may be attributed to both biologic and behavioural factors. ${ }^{17}$ Some studies have identified caregiving and family commitments as barriers to physical activity among women. ${ }^{18}$ The prevalence of ideal cardiovascular health declined with age. Weight gain $^{19}$ and the increasing prevalence of hyperten$\operatorname{sion}^{20}$ and diabetes ${ }^{13}$ with age have been noted by other studies and are likely to contribute to this relation. The slight increase in the prevalence of ideal cardiovascular health among men at older ages may stem from a survivorship effect (men in better cardiovascular health tend to survive to older ages). We found a strong gradient of increasing cardiovascular health from the Eastern to Western provinces. Similar patterns have been observed for ischemic heart disease and cardiovascular disease mortality. ${ }^{21}$ Interprovincial differences that may contribute to this gradient include age, socioeconomic factors (e.g., education, income, employment status), the ethnic makeup of populations and community characteristics (e.g., availability of recreational facilities and healthy foods).

We found that fewer than 1 in 10 adults and 1 in 5 youth met the criteria for ideal cardiovascular health. To improve the cardiovascular health of Canadians, the Heart and Stroke Foundation of Canada recently developed 2 goals: improve the cardiovascular health of Canadians by $10 \%$ by 2020 (compared with baseline years 2009-2010) as measured with the CANHEART health index, and reduce cardiovascular mortality rates in Canada by $25 \%$ by 2020 . To achieve these goals, the Canadian Heart and Stroke Foundation will develop health promotion strategies aimed at improving population levels of the health behaviours and factors that make up the CANHEART health index. However, changing health behaviours and factors at a societal level is difficult and will require a concerted multipronged approach.

\section{Limitations}

Our study has some limitations that should be considered. All CCHS survey data was selfreported; however, we do not believe this to be a major limitation because previous validation work has shown self-reported hypertension and diabetes in the CCHS to have good agreement with validated case definitions derived by use of administrative data. ${ }^{22}$ In addition, self-reported smoking status shows strong agreement with uri-

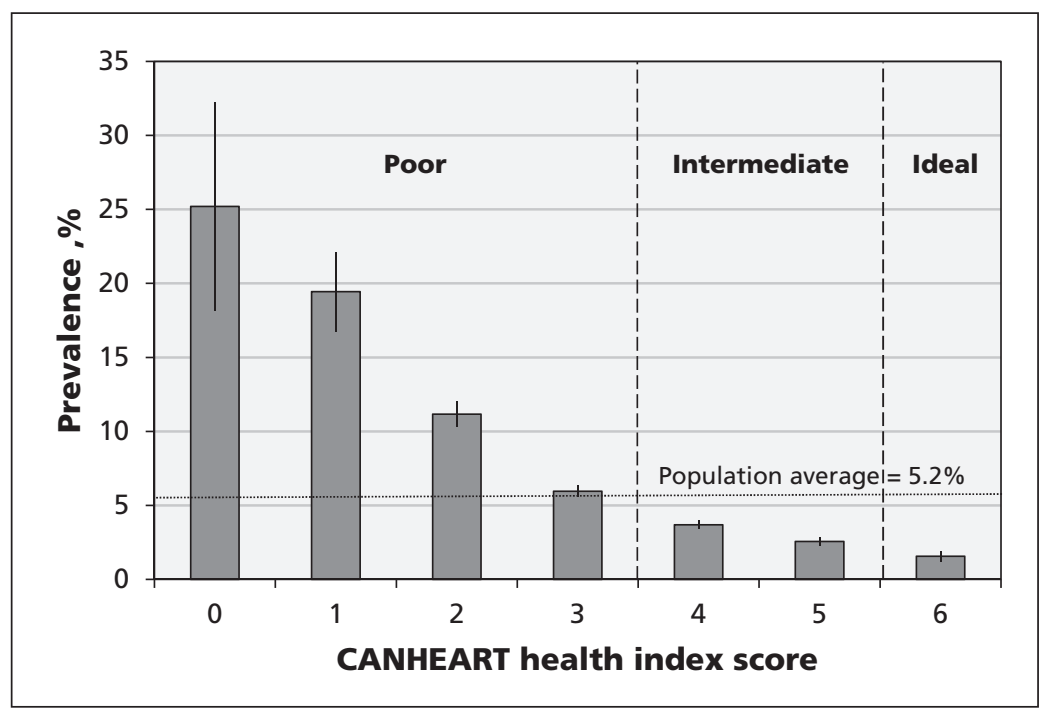

Figure 5: Weighted prevalence of heart disease among adults age 20 and older by CANHEART health index score. Error bars indicate $95 \%$ confidence intervals. Among adults, ideal cardiovascular health is defined as the presence of all 6 ideal health factors and behaviours (smoking status, overweight/obesity, physical activity, fruit and vegetable consumption, hypertension, diabetes); intermediate cardiovascular health is defined as 4-5 ideal health factors or behaviours; and poor cardiovascular health is defined as the presence of 0-3 ideal health factors or behaviours. Source: Canadian Community Health Survey 2009-2010. 
nary cotinine concentration. ${ }^{23}$ Because we did not have access to detailed clinical and anthropometric measurements (e.g., blood pressure, glucose, cholesterol levels) or measured height or weight, we were unable to include these in our definition of ideal cardiovascular health. Data from the Canadian Health Measures Survey (2007-2009) indicates that $41 \%$ of Canadians aged 20-79 years had total cholesterol levels of $5.2 \mathrm{mmol} / \mathrm{L}$ or higher, suggesting that our study may have overestimated the number of Canadians in ideal cardiovascular health. ${ }^{24}$ Information on important dietary factors (e.g., sodium, fats, whole grains, fish) and total caloric intake were not available in the CCHS; thus, our dietary measure may not have been sufficiently detailed to capture the complete effect of diet on cardiovascular health. The cross-sectional nature of the survey data precluded examination of changes in the health behaviours and health status of individuals over time, including behavioural changes that may stem from disease diagnoses.

\section{Conclusion}

Our study has shown important trends in health behaviours and factors related to the development of cardiovascular disease in Canadian adults and youth. Although the levels of physical activity, fruit and vegetable consumption, and smoking appear to be improving, worsening levels of obesity, hypertension and diabetes require further attention. Building on the positive changes seen for some health behaviours, targeted policy and program interventions are needed to increase all types of physical activity and dietary quality, which will improve the cardiovascular health of Canadians and decrease the incidence of cardiovascular disease. Fewer than 1 in 10 Canadian adults and 1 in 5 youth were in ideal cardiovascular health during the study period; the prevalence of ideal cardiovascular health has remained stable in adults since 2003 and has shown moderate improvement in youth.

We anticipate that the CANHEART health index will be a useful tool for members of the general public, clinicians, researchers, chronic disease organizations and decision-makers interested in monitoring cardiovascular health and reducing the burden of chronic noncommunicable diseases in Canada.

\section{References}

1. Tracking heart disease and stroke in Canada. Ottawa (ON): Public Health Agency of Canada; 2009. Available: www.phac-aspc .gc.ca/publicat/2009/cvd-avc/pdf/cvd-avs-2009-eng.pdf (accessed 2013 Oct. 25)

2. Lloyd-Jones DM, Hong Y, Labarthe D, et al. Defining and setting national goals for cardiovascular health promotion and disease reduction: the American Heart Association's strategic impact goal through 2020 and beyond. Circulation 2010;121:586-613.
3. Folsom AR, Yatsuya H, Nettleton JA, et al. Community prevalence of ideal cardiovascular health, by the American Heart Association definition, and relationship with cardiovascular disease incidence. J Am Coll Cardiol 2011;57:1690-6.

4. Zhang Q, Zhou Y, Gao X, et al. Ideal cardiovascular health metrics and the risks of ischemic and intracerebral hemorrhagic stroke. Stroke 2013;44:2451-6.

5. Yang Q, Cogswell ME, Flanders WD, et al. Trends in cardiovascular health metrics and associations with all-cause and CVD mortality among US adults. JAMA 2012;307:1273-83.

6. Rasmussen-Torvik LJ, Shay CM, Abramson JG, et al. Ideal cardiovascular health is inversely associated with incident cancer clinical perspective: the Atherosclerosis Risk in Communities study. Circulation 2013;127:1270-5.

7. Canadian Community Health Survey annual component. Ottawa $(\mathrm{ON})$ : Statistics Canada; 2012. Available: www23.statcan .gc.ca/imdb/p2SV.pl?Function=getSurvey\&SDDS=3226\&lang=en $\& d b=i m d b \& a d m=8 \& d i s=2$ (accessed 2013 Aug. 30).

8. Cole TJ, Bellizzi MC, Flegal KM, et al. Establishing a standard definition for child overweight and obesity worldwide: international survey. BMJ 2000;320:1240-3.

9. World Health Organization Expert Consultation. Appropriate body-mass index for Asian populations and its implications for policy and intervention strategies. Lancet 2004;363:157-63

10. Canadian physical activity guidelines and Canadian sedentary behaviour guidelines. Ottawa (ON): Canadian Society for Exercise Physiology; 2013. Available: www.csep.ca/english/view .asp? $\mathrm{x}=804$ (accessed 2013 Aug. 30).

11. Bélanger-Ducharme F, Tremblay A. Prevalence of obesity in Canada. Obes Rev 2005;6:183-6.

12. Tu K, Chen Z, Lipscombe LL. Prevalence and incidence of hypertension from 1995 to 2005: a population-based study. CMAJ 2008;178:1429-35.

13. Lipscombe LL, Hux JE. Trends in diabetes prevalence, incidence, and mortality in Ontario, Canada 1995-2005: a population-based study. Lancet 2007;369:750-6.

14. Global health risks. Geneva (Switzerland): World Health Organization; 2009. Available: www.who.int/healthinfo/global_burden _disease/GlobalHealthRisks_report_full.pdf (accessed 2013 Oct. 25).

15. Tu JV, Nardi L, Fang J, et al. National trends in rates of death and hospital admissions related to acute myocardial infarction, heart failure and stroke, 1994-2004. CMAJ 2009;180:E118-25.

16. Yusuf S, Hawken S, Ounpuu S, et al. Effect of potentially modifiable risk factors associated with myocardial infarction in 52 countries (the INTERHEART study): case-control study. Lancet 2004;364:937-52.

17. Pilote L, Dasgupta K, Guru V, et al. A comprehensive view of sex-specific issues related to cardiovascular disease. CMAJ 2007; 176:S1-44.

18. King AC, Castro C, Wilcox S, et al. Personal and environmental factors associated with physical inactivity among different racial-ethnic groups of US middle-aged and older-aged women. Health Psychol 2000;19:354-64.

19. Rosengren A, Wedel H, Wilhelmsen L. Body weight and weight gain during adult life in men in relation to coronary heart disease and mortality: a prospective population study. Eur Heart J 1999; 20:269-77.

20. Robitaille C, Dai S, Waters C, et al. Diagnosed hypertension in Canada: incidence, prevalence and associated mortality. CMAJ 2012;184:E49-56.

21. Filate WA, Johansen HL, Kennedy C, et al. Regional variations in cardiovascular mortality in Canada. Can J Cardiol 2003;19:1241-8.

22. Muggah E, Graves E, Bennett C, et al. Ascertainment of chronic diseases using population health data: a comparison of health administrative data and patient self-report. BMC Public Health 2013;13:16

23. Assessment of validity of self-reported smoking status. Ottawa (ON): Statistics Canada; 2012. Available: www.statcan.gc.ca /pub/82-003-x/2012001/article/11625-eng.pdf (accessed 2013 Aug. 30).

24. Heart health and cholesterol levels of Canadians, 2007 to 2009. Ottawa (ON): Statistics Canada; 2010. Available: www.statcan .gc.ca/pub/82-625-x/2010001/article/11136-eng.htm (accessed 2013 Oct. 25).

Affiliations: Institute for Clinical Evaluative Sciences (Maclagan, Manuel, Gershon, Booth, Bhatia, Atzema, Tu), Toronto, Ont.; Statistics Canada (Park, Sanmartin), Ottawa, Ont.; Heart and Stroke Foundation of Canada (Mathur, Roth), Toronto, Ont.; Ottawa Hospital Research Institute (Manuel), Ottawa, Ont.; St. Michael's Hospital (Booth), Toronto, Ont.; Women's College Hospital Institute for Health 
Systems Solutions and Virtual Care (Bhatia), Toronto, Ont.; Department of Medicine (Booth, Tu), University of Toronto, Toronto, Ont.; Sunnybrook Health Sciences Centre (Gershon, Atzema), Toronto, Ont.; Schulich Heart Centre (Tu), Sunnybrook Health Sciences Centre, Toronto, Ont.

Contributions: Jack Tu conceptualized and designed the study. Jungwee Park and Laura Maclagan conducted the statistical analysis. Jungwee Park, Claudia Sanmartin and Douglas Manuel provided methodologic advice. Laura Maclagan completed the literature review and drafted the manuscript. All authors contributed to the analysis and interpretation of the data, revised the manuscript for intellectual content and approved the final version submitted for publication

Funding: This study was supported by an operating grant from the Institute for Circulatory Health-Canadian Institutes of Health Research Chronic Diseases Team (grant no. TCA 118349) to the Cardiovascular Health in Ambulatory Care Research Team (CANHEART) and by the Heart and
Stroke Foundation of Canada. Additional support was provided by the Institute for Clinical Evaluative Sciences (ICES), which is funded by an annual grant from the Ontario Ministry of Health and Long-Term Care (MOHLTC). No endorsement by ICES or the Ontario MOHLTC is intended or should be inferred. Jack Tu is supported by a Canada Research Chair in Health Services Research and a Career Investigator Award from the Heart and Stroke Foundation of Ontario. The results and conclusions are those of the authors and should not be attributed to any of the funding agencies or agencies whose data was used in the study.

Acknowledgements: We thank Drs. Donald Lloyd-Jones and Mark Huffman, Northwestern University Feinberg School of Medicine, for providing additional background information on the American Heart Association's definition of ideal health and methodologic advice about the development of the CANHEART health index. Additional information about the CANHEART health index can be obtained by visiting the CANHEART study website (www.canheart.ca).

\section{How you can get involved in the CMA!}

The CMA is committed to providing leadership for physicians and promoting the highest standard of health and health care for Canadians. To strengthen the association and be truly representative of all Canadian physicians the CMA needs to hear from members interested in serving in elected positions and on appointed committees and advisory groups. The CMA structure comprises both governing bodies and advisory bodies either elected by General Council or appointed by the CMA Board of Directors. The Board of Directors - elected by General Council — has provincial/territorial, resident and student representation, is responsible for the overall operation of the CMA and reports to General Council on issues of governance.

CMA committees advise the Board of Directors and make recommendations on specific issues of concern to physicians and the public. Five core committees mainly consist of regional, resident and student representation while other statutory and special committees and task forces consist of individuals with interest and expertise in subject-specific fields. Positions on one or more of these committees may become available in the coming year.

For further information on how you can get involved please go to http://www.cma.ca/membercentre/how-you-can-get-involved, or contact

\section{Cherise Araujo \\ Corporate and Governance Services \\ Canadian Medical Association \\ 1867 Alta Vista Drive, Ottawa ON K1G 5 W8 \\ Fax 613 526-7570, Tel 800 663-7336 x1949 \\ cherise.araujo@cma.ca}

By getting involved, you will have an opportunity to make a difference.

We hope to hear from you!

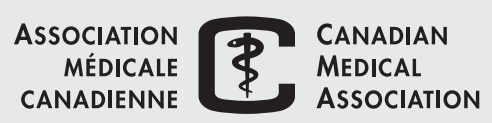

